\title{
Experimental characterization and macro-modeling of mechanical strength of multi-sheets and multi-materials spot welds under pure and mixed modes I and II
}

\author{
Rim Chtourou ${ }^{1,2, a}$, Gregory Haugou ${ }^{1, b}$, Nicolas Leconte ${ }^{1, \mathrm{c}}$, Bassem Zouari ${ }^{2, \mathrm{~d}}$, Fahmi Chaari ${ }^{1, \mathrm{e}}$, and Eric Markiewicz ${ }^{1, \mathrm{f}}$ \\ ${ }^{1}$ LAMIH, UMR CNRS 8201, University of Valenciennes, 59313 Valenciennes Cedex 9, France \\ ${ }^{2}$ LA2MP, National Engineering School of Sfax (ENIS), Route de Soukra, BP. W, 3038 Sfax, Tunisia
}

\begin{abstract}
Resistance Spot Welding (RSW) of multiple sheets with multiple materials are increasingly realized in the automotive industry. The mechanical strength of such new generation of spot welded assemblies is not that much dealt with. This is true in particular for experiments dedicated to investigate the mechanical strength of spot weld made by multi sheets of different grades, and their macro modeling in structural computations. Indeed, the most published studies are limited to two sheet assemblies.

Therefore, in the first part of this work an advanced experimental set-up with a reduced mass is proposed to characterize the quasi-static and dynamic mechanical behavior and rupture of spot weld made by several sheets of different grades. The proposed device is based on Arcan test, the plates contribution in the global response is, thus, reduced. Loading modes I/II are, therefore, combined and well controlled.

In the second part a simplified spot weld connector element (macroscopic modeling) is proposed to describe the nonlinear response and rupture of this new generation of spot welded assemblies. The weld connector model involves several parameters to be set. The remaining parameters are finally identified through a reverse engineering approach using mechanical responses of experimental tests presented in the first part of this work.
\end{abstract}

\section{Introduction}

Resistance spot welding (RSW) is the most common joining process for automotive body-in-white structures due to its production convenience and cost effectiveness. Around four thousands spot welds are indeed employed to assemble the body-in-white. With the demand of safety and lightweight vehicle structures, RSW of multiple sheets (more than two) and combining multiple materials (i.e. different steel grades) are increasingly realized. The literature survey shows that the mechanical strength of two sheet spot welded assemblies has been widely studied experimentally and numerically.

On the one hand, many tests are used, like pull-out, coach-peel and single-lap shear samples under quasi-static and dynamic loading conditions. Since 1958, mechanical tests have been discussed [1-3] in particular for pure tension, tension-shear and peel specimens. For this type of tests, a large part of the sheet sample plastically deforms, in particular far from the spot weld. Thus, it contributes significantly in the global behavior. Moreover, it is difficult to express the failure properties because the tensile/shear load ratio conditions varies during the test. More recently, many researchers [4-8] have proposed to adapt the Arcan tests to investigate the failure characteristics of spot

\footnotetext{
a e-mail: Rim.Chtourou@etu.univ-valenciennes-.fr

b e-mail: gregory.haugou@univ-valenciennes. fr

c e-mail: Nicolas.Leconte@univ-valenciennes.fr

d e-mail: bzouari@yahoo.com

e e-mail: fahmi.chaari@univ-valenciennes.fr

f e-mail: eric.markiewicz@univ-valenciennes.fr
}

welds under combined loading conditions. Indeed, Arcan tests have advantages over standard tests that the plates contribution around the weld nugget in the mechanical response is reduced. Moreover, it allows tensile (mode I) and shear (mode II) loads to be pure or combined and well controlled. However, these devices are characterized by a large mass that generates inertial forces during the dynamic loading. Thus, they can be used only under quasistatic loading conditions. To correct this limitation and to investigate the strain rate sensitivity of spot welds in dynamic conditions, Langrand and Markiewicz [7] have proposed an experimental device with a reduced mass to cope with these inertial forces.

On the other hand, researchers have developed various models to investigate the mechanical strength of spot welds. 3D FE detailed mesoscopic models allow a good representation of the nonlinear behavior of the assembly [9-11]. But such models are very expensive in terms of calculation time and are more dedicated to preliminary study, not for structural computations. Therefore, simplified models of the spot weld behavior and rupture are required. Some macro models have been developed and published in the literature [12-15]. One of the most interesting is that of Combescure et al. $[6,13]$, which is an elastic-plastic damageable Finite Element (FE) that models the global mechanical behavior of the whole "welded zone".

Almost all existing models have been evaluated for two-sheet assemblies. However, their performance to model multi-sheet multi-material spot welded assemblies remains challenging. Moreover, to the knowledge of the 


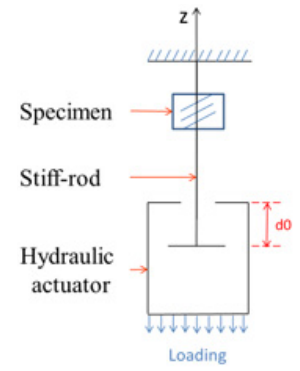

(a)

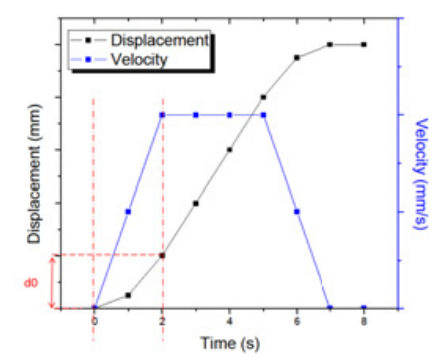

(b)
Figure 1. (a) Scheme of loading conditions (b) Typical curve of the displacement of the actuator.

authors, spot welds strength experiments that can be found in the literature deal only with two-sheet assemblies.

As a first part of this work an advanced experimental procedure is proposed to characterize the quasi-static and dynamic behavior of multi-sheet and multi-steel grades spot welds under pure and combined modes I/II loading conditions. An original experimental device based on the Arcan principle is designed to characterize specimens that consist of three-sheet spot welded assemblies. The second part of the work deals with the formulation of a connector model to simulate the global behavior and failure of spot welded assemblies. Following the modeling of this kind of assembly, the parameters of the joint equivalent model are identified based on the experimental results of the presented experiments.

Finally, some conclusions are presented.

\section{New advanced experimental procedures}

\subsection{Experimental device}

The proposed new device is designed to minimize inertia effects (optimized geometry and titanium alloy used as constitutive material). It allows to investigate the mechanical strength of these three-sheets assemblies under pure and combined modes I/II dynamic loading conditions. Note that an adequate test device was especially designed for each angular position.

The tests are carried out on a high speed hydraulic machine (INSTRON VHS 65/20). The hydraulic actuator moves along vertical axis ( $\mathrm{z}$ ) with an imposed speed to load the specimen. Under dynamic conditions, the displacement of the actuator increases progressively with the time until a linear regime. To ensure a constant speed loading during the tensile test, an experimental arrangement has been designed such as only the actuator moves without any loading of the sample along a distance a distance called here $d_{0}$ (Fig. 1(a)). Once the speed in established and the loading velocity becomes constant, the contact is effected, then the stiff-rod and the specimen are loaded as presented in Fig. 1(b).

This device allows tensile and shear loads to be combined and controlled. The angular position $\alpha$ between the loading direction (the vertical axis) and the normal to

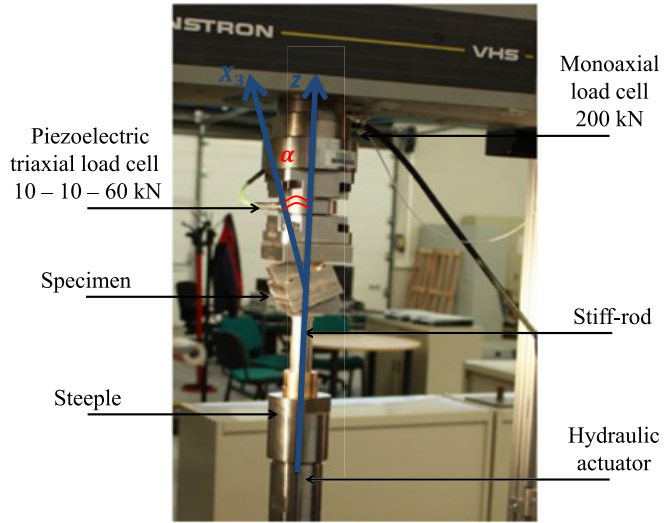

Figure 2. Experimental device for three-sheets assembly tensile testing.

Table 1. Mechanical properties of sheets metals.

\begin{tabular}{lclc}
\hline & $\sigma_{y}(M P a)$ & $\sigma_{\max }(M P a)$ & $A(\%)$ \\
\hline$D X 54 D$ & 171 & 301.6 & 57 \\
$22 M n B 5$ & 1100 & 1500 & 6 \\
\hline
\end{tabular}

the surface of the specimen defines the tensile/shear ratio (Fig. 2).

The device was fixed to the machine with four screws. All device rotations are canceled. Forces along the three main directions of the machine $\left(F_{x}, F_{y}\right.$ and $\left.F_{z}\right)$ are measured during the tests, using a triaxial load cell (Kistler 9367C). The displacement is obtained by a LVDT sensor with a measurement range equal to $300 \mathrm{~mm}$ and error of $0.15 \mathrm{~mm}$. Figure 2 shows the complete set up for the experiments.

\subsection{Three-sheets spot weld specimen based on two constitutive materials}

The proposed specimen consist of a three-sheets assembly: $\left(P_{1}\right) 2 \mathrm{~mm}$ thick $22 \mathrm{MnB} 5,\left(P_{2}\right) 0.65 \mathrm{~mm}$ thick DX54D and $\left(P_{3}\right) 1.6 \mathrm{~mm}$ thick $22 \mathrm{MnB} 5$ (Fig. 3). The spot welding process was performed by our industrial partner Renault according to the know-how of the manufacturer. $22 \mathrm{MnB} 5$ alloy is an ultra high strength steel, and it is the base material for the two external plates of the assembly. The middle plate is made of mild steel (DX54D). The mechanical properties of the two materials are presented in Table 1.

As it is shown in Fig. 3, two rigid heel joints have been designed similarly to the set up proposed by Langrand and Markiewicz [7] to ensure the connection between the specimen and the device. The assembly, rigid heel joint and specimen, is positioned on the device by two centering pins and fixed with six screws. The set-up is designed to limit the bending of sheets and ensure a good repeatability of the tests. The free part around the spot weld is circular for isotropy effect.

With three-sheets assemblies, three loading configurations (1-3), (1-2) and (2-3) are possible. For the first part of this project, only the configuration (1-3), which consist of loading the first and the third sheet of the assembly, has been studied and presented in this paper. Two other 


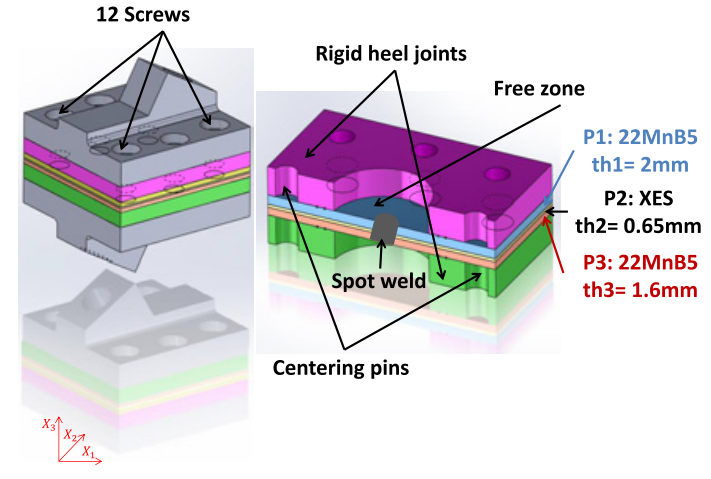

Figure 3. Three-sheets spot welds specimens based on two constitutive materials (22MnB5 and DX54D).

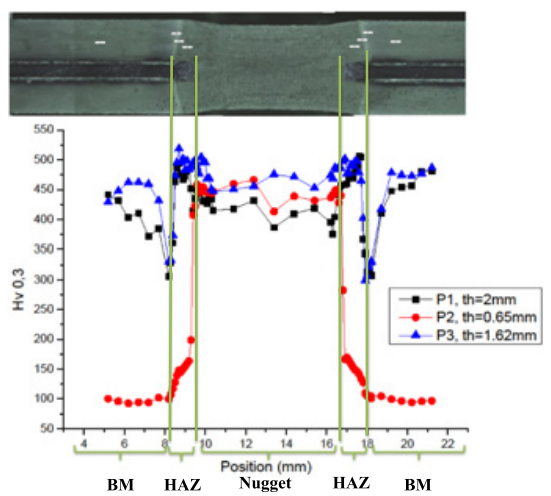

Figure 4. Hardness distribution along the diametrical direction of manufactured spot weld joint.

configurations (1-2) and (2-3) are currently in progress, and their results will be subject of future publications.

\subsection{Hardness measurement}

Vickers hardness testing was performed with Future Tech Hardness Tester (Model FM). The applied force level for hardness measurement was equal to $300 \mathrm{~g}$. The hardest zone of the test samples (spot weld nugget) is indented with a pitch of $0.1 \mathrm{~mm}$ and the softest zone (base metal) parts indented with a pitch of $1 \mathrm{~mm}$.

The averaged and linearised hardness distribution results revealed the nugget dimensions, and allows us to identify three zones of spot weld: Nugget, Heat Affected Zone (HAZ) and Base Material (BM) (Fig. 4). The shape of the nugget will be used for the numerical modeling which is presented in the later section.

\subsection{Experimental results}

To study the strain-rate sensitivity of spot welds, three velocities are employed in this work: $\mathrm{v}=[1 \mathrm{~mm} / \mathrm{s}$, $33 \mathrm{~mm} / \mathrm{s}, 1 \mathrm{~m} / \mathrm{s}$ ]. So that, a jump of strain rate close to 30 is attended. For each loading speed four loading directions are chosen: pure tensile (mode I) with $\alpha=0^{\circ}$, pure shear (mode II) with $\alpha=90^{\circ}$, and combined loading with $\alpha=25^{\circ}$ and $\alpha=45^{\circ}$. A set of two or three tests has been performed for each angle/velocity combination.
Table 2. Experimental results of the different loading conditions.

\begin{tabular}{lllll}
\hline & & $1 \mathrm{~mm} / \mathrm{s}$ & $33 \mathrm{~mm} / \mathrm{s}$ & $1 \mathrm{~m} / \mathrm{s}$ \\
\hline$\alpha=0^{\circ}$ & $F_{\text {max }}(\mathrm{kN})$ & 13.6 & 12.7 & 14.3 \\
& $U_{\text {rup }}(\mathrm{mm})$ & 1.83 & 2.27 & 2.2 \\
\hline$\alpha=25^{\circ}$ & $F_{\text {max }}(\mathrm{kN})$ & 13.8 & 13.7 & 14.2 \\
& $U_{\text {rup }}(\mathrm{mm})$ & 1.52 & 1.85 & 2 \\
\hline$\alpha=45^{\circ}$ & $F_{\text {max }}(\mathrm{kN})$ & 15.1 & 16.2 & - \\
& $U_{\text {rup }}(\mathrm{mm})$ & 1.5 & 1.82 & - \\
\hline$\alpha=90^{\circ}$ & $F_{\text {max }}(\mathrm{kN})$ & 38.8 & - & - \\
& $U_{\text {rup }}(\mathrm{mm})$ & 2.18 & - & - \\
\hline
\end{tabular}

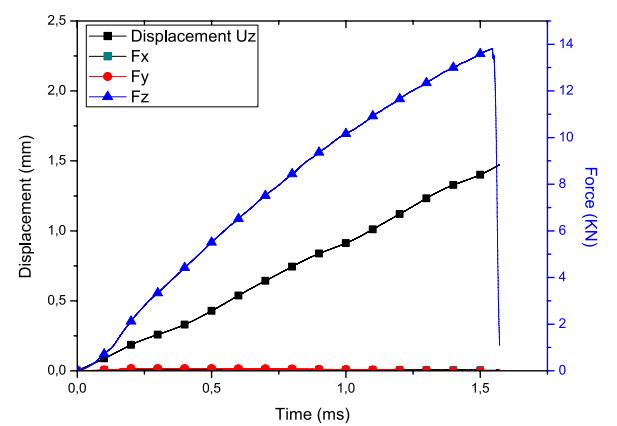

Figure 5. Typical forces and displacement versus time curves at $1 \mathrm{~mm} / \mathrm{s}$ under pure tension loading.

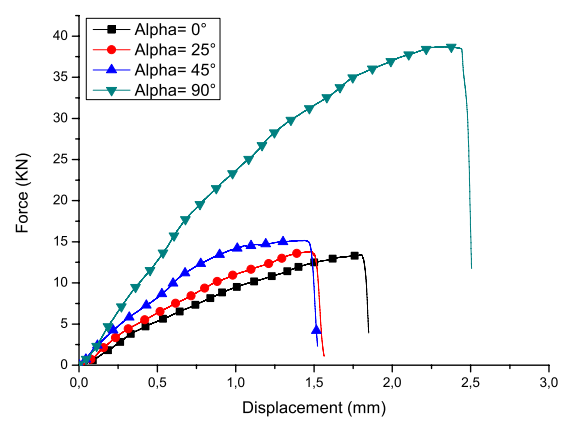

Figure 6. Typical force versus displacement diagrams measured under quasi-static $(V=1 \mathrm{~mm} / \mathrm{s})$ pure and combined tensile/shear modes.

\subsubsection{Influence of the load angle at a constant speed}

Figure 5 shows typical curves of forces and displacement versus time at $1 \mathrm{~mm} / \mathrm{s}$ under pure tension loading. As it is expected, the displacements increase linearly during loading, thus, the speed remains constant during the test. Also, tangential forces $\left(F_{x}\right.$ and $\left.F_{y}\right)$ are negligible compared to the normal force $F_{z}$ (loading direction).

Figure 6 shows typical experimental results of force versus displacement under different loading angles for a velocity at $1 \mathrm{~mm} / \mathrm{s}$. The different forces versus elongation responses have the same shape. Unlike the case of twosheet assemblies [7], the failure occurs suddenly and there is no dissipated energy after the maximum load, for the shear test and the combined loading. That might be due to the fact that the tests are carried out in configuration (1-3). Indeed, the loading is applied to the plates made on $22 \mathrm{MnB5}$. Therefore, other failure modes can be detected for configurations (1-2) and (2-3). 


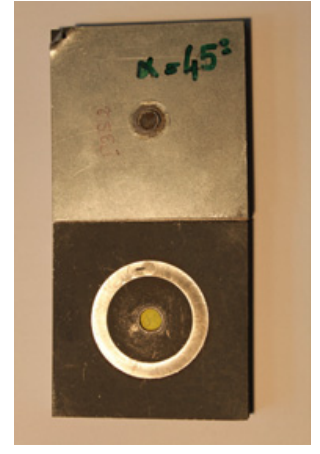

(a)

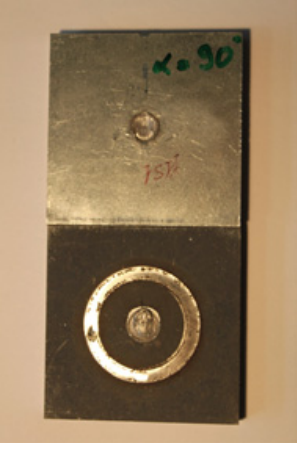

(b)
Figure 7. Failure mode (a) $\alpha=45^{\circ}$ (b) $\alpha=90^{\circ}$.

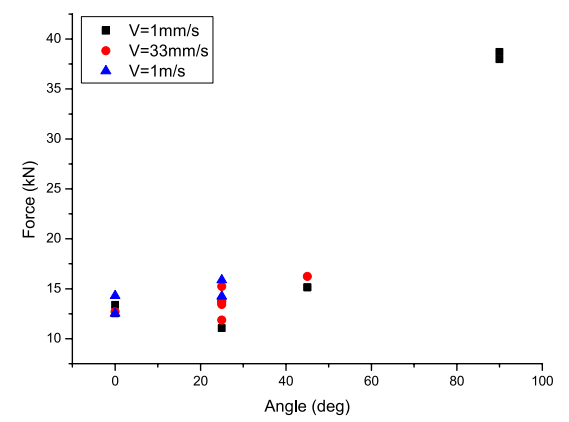

Figure 8. Evolution of the maximal load with the variation of the loading angle.

Note that, the maximum loading force is quasi-constant for $\alpha=0^{\circ}$ to $\alpha=45^{\circ}$ (mean value $F_{\text {max }}=15 \mathrm{kN}$ ), but brutally increases up to $F_{\max }=39 \mathrm{kN}$ for $\alpha=90^{\circ}$ (Fig. 8). This is because for loading angle between $\alpha=0^{\circ}$ and $\alpha=45^{\circ}$ the failure occurs suddenly when the metal tears at once all around the welded zone (Fig. 7(a)). However for shear test $\left(\alpha=90^{\circ}\right)$ the core of the spot weld itself has been cutted off as it is shown in Fig. 7(b). Moreover, spot weld core is characterized by the higher hardness of the assembly because of the thermal treatment during the welding process.

Concerning ultimate elongation, its evolution is not perfectly established due to repeatability issues and centering defaults of the spot weld detected in the free zone (Fig. 3).

\subsubsection{Strain-rate effect}

To study the strain-rate effect, Table 2 summarize the evolution of the maximum loading force and the the motion at the rupture with variation of the loading speed. In fact, the maximum loading force increases slightly with velocity. For the case of loading angle $\alpha=0^{\circ}$, it increase by $5 \%$ between the lower and the higher velocity and by $2.8 \%$ for $\alpha=25^{\circ}$. For the case $\alpha=45^{\circ}$ we observe that the maximum load in the case of $33 \mathrm{~mm} / \mathrm{s}$ velocity is lower of $7 \%$ than the case of $1 \mathrm{~mm} / \mathrm{s}$ velocity. The displacement at the break increases, also, by $20 \%$ for the different loading angle Fig. 9 shows the evolution of the displacement at the break with the loading speed. Indeed, when the velocity increases from $1 \mathrm{~mm} / \mathrm{s}$ to $33 \mathrm{~mm} / \mathrm{s}$.

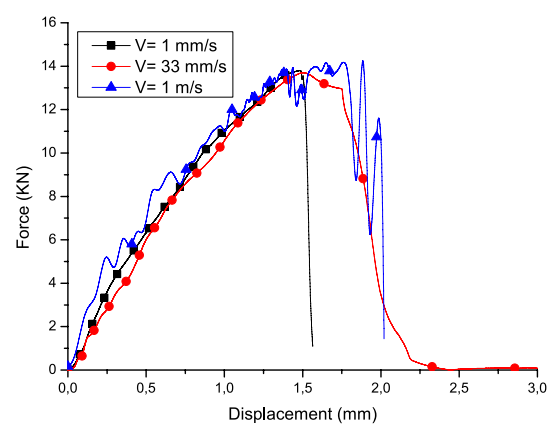

Figure 9. Influence of the loading speed in combining loading $\alpha=25^{\circ}$.

Table 3. Evolution of the dissipated energy.

\begin{tabular}{lccc}
\hline & $\mathrm{V}=1 / \mathrm{V}=33$ & $\mathrm{~V}=33 / \mathrm{V}=1000$ & $\mathrm{~V}=1 / \mathrm{V}=1000$ \\
\hline$\alpha=0^{\circ}$ & $38 \%$ & $1.5 \%$ & $36 \%$ \\
\hline$\alpha=25^{\circ}$ & $37 \%$ & $8 \%$ & $51 \%$ \\
\hline$\alpha=45^{\circ}$ & $43 \%$ & - & - \\
\hline
\end{tabular}

The augmentation of the loading speed generates an increasing of the maximum loading force and the displacement at the break and consequently an increasing of the dissipated energy. Therefore, the energy dissipation is strain rate dependent. Table 3 represents the evolution of energy dissipation for each jump of strain, for different loading angles. The obtained results proves that the energy dissipation strain rate dependency is more important when the loading angle is larger.

Such results can't be yet confirmed because of the lack of experimental results. In the same way, any conclusion can be done for shear tests experiments.

Other experimental tests with other intermediate loading angle $\left(\alpha=60^{\circ}\right.$ and $\left.\alpha=75^{\circ}\right)$ will be done to detect a representative trend for the evolution of the ultimate displacement and force with the loading angle. These complementary tests will also give information about the strain rate effect.

These results will be used to identify the parameters of the macro modeling approach presented in the following section.

\section{Connector model formulation}

The spot weld connector is modeled by an equivalent element featuring two nodes, similar to a generalized spring where the degrees of freedom are the relative generalized motion of referential to another.

As the local behavior of a spot weld is nonlinear, an elastic-plastic model has been introduced to define the mechanical behavior of the spot weld connector.

Stiffnesses of the spot weld connector model are assumed to be uncoupled, $\left\{f_{i}\right\}=\left[D_{i i}\right]\left\{u_{i}\right\}$.

The plasticity criterion is expressed by Eq. (1).

$$
\phi\left(\bar{F}, \bar{u}^{p l}\right)=\bar{F}-F_{H} \leq 0
$$

where $\phi\left(\bar{F}, \bar{u}^{p l}\right)$ is the yield surface of the plastic model, $\bar{F}$ is the equivalent generalized force of spot weld connector and $F_{H}$ is the hardening function. $\bar{F}$ is calculated using the 


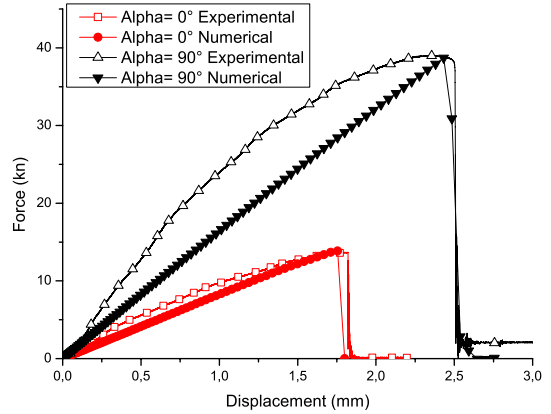

Figure 10. Load vs. displacement diagrams measured underpure tensile mode $\alpha=0^{\circ}$.

potential function $P_{1}(2)$.

$$
\begin{aligned}
\bar{F}= & P_{1}(\{f\})=\left[\left(\frac{F_{N}}{R_{N}}\right)^{\beta}+\left(\frac{F_{S}}{R_{S}}\right)^{\beta}\right. \\
& \left.+\left(\frac{M_{T}}{R_{T}}\right)^{\beta}+\left(\frac{M_{B}}{R_{B}}\right)^{\beta}\right]^{\frac{1}{\beta}}
\end{aligned}
$$

where the $F_{N}$ is normal force, $F_{S}$ the shear force, $M_{B}$ the bending moment, and $M_{T}$ torsion moment.

Parameters $\beta, R_{N}, R_{S}, R_{B}$ and $R_{T}$ are chosen to fit the plastic behavior under different loading directions.

For this model an exponential Voce type law is chosen to model the isotropic hardening behavior of the spot weld connector model (Eq. (3)).

$$
F_{H}=F_{0}+Q\left(1-\exp \left(-b \bar{u}^{p l}\right)\right)
$$

where $F_{0}$ is the yield value at zero plastic equivalent motion, $Q$ is the saturation generalized force and $b$ is a constant.

The failure of the connection is taken into account by a ductile damage model.

Damage is initiated when $\left(\bar{F} \geq F_{i}\right)$, where $F_{i}$ the critical force value. The damage evolution is defined by linear law that specifies how the damage variable $d$ evolves. Then, the force response is expressed by Eq. (4):

$$
\bar{F}_{d}=(1-d) \bar{F}, 0 \leqslant d \leqslant 1 .
$$

The set of parameters, which describe the elasto-plastic damage behavior of the spot weld colled, are called design parameters, have to be identified by reverse engineering approach.

\section{Numerical modeling and results}

The three-sheets two-materials spot weld assembly presents very big metallurgical and structural complexities. Therefore, different modeling methods can be proposed. In this work, the three-sheets spot welded assembly was modeled by two connector elements in series (Sect. 3). The same mechanical behavior was associated for the two connectors. Thus, just one set of parameters will be identified for the two connectors.

According to the description of the proposed experimental set-up, the spot-welded specimens are modeled by

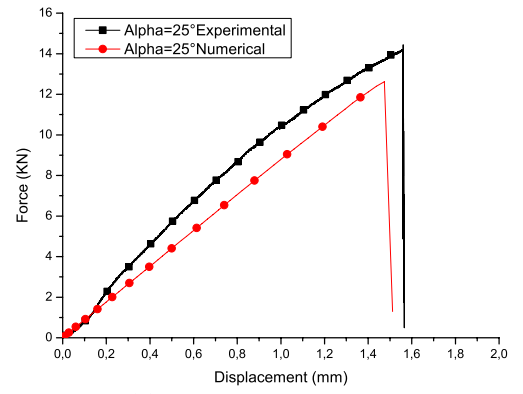

(a)

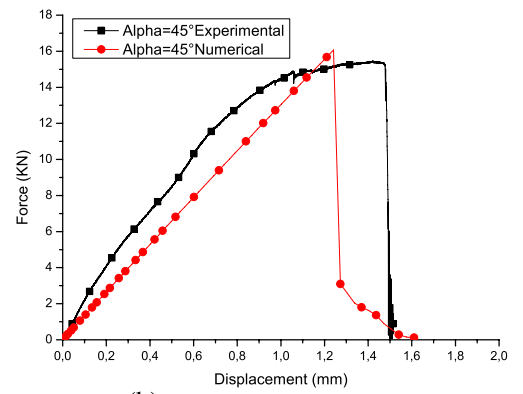

(b)

Figure 11. Load vs. displacement diagrams measured under combined tensile/shear mode(a) $\alpha=25^{\circ}$ (b) $\alpha=45^{\circ}$.

Table 4. Comparaison of total dissipated energy at rupture betwen numerical and experimental results.

\begin{tabular}{lllll}
\hline$\alpha^{\circ}$ & 0 & 25 & 45 & 90 \\
\hline Error in energy (\%) & 15.6 & 21.1 & 30 & 22.1 \\
\hline
\end{tabular}

three parallel circular plates of $20 \mathrm{~mm}$ diameter each one corresponding to the free part sheet metal around the spot weld. The three plates are meshed with quadrangular shell elements of $8 \mathrm{~mm}$ size, type S4R with a reduce integration. The periphery of the top plate is defined as a rigid body. The extremity of the bottom plate is clamped and an imposed velocity $V_{\mathrm{imp}}=1 \mathrm{~mm} / \mathrm{s}$ is applied to the rigid body according to mode I and mode II directions (quasistatic loading).

The maximum force and the displacement at failure are very well described for the tensile test (mode I) and for shear test (mode II) (Fig. 10).

For the model validation, it is proposed a comparison of the experimental and numerical simulation results under mixed loads. Figure 1(a) and Fig. 1(b) present a comparison of the model results to experimental ones for angular positions $\alpha=25^{\circ}$ and $\alpha=45^{\circ}$, respectively.

Satisfactory results are obtained. The maximum loading force is underestimated by $10 \%$ for $\alpha=25^{\circ}$. However, for $\alpha=45^{\circ}$ it is overestimated by $7 \%$.

In terms of energy, Table 4 summarizes the energy error for different loading angles.

\section{Conclusion}

An experimental procedure has been proposed in order to investigate the mechanical behavior of multi-materials multi-sheets spot welded assemblies under quasi-static and dynamic loading conditions and in pure and combined opening/shear modes. The experimental set-up is based on 
the Arcan test principle, with a reduced mass to investigate the strain rate of this type of assembly. A first part of the experimental tests has been done in four loading directions and for three loading velocities, and preliminary results have been obtained. Other experimental tests with other intermediate loading angle $\left(\alpha=60^{\circ}\right.$ and $\left.\alpha=75^{\circ}\right)$ and two other configurations will be done to detect a trend for the evolution of the ultimate displacement and define a failure criteria.

Authors have also proposed to study numerically the nonlinear mechanical behavior of the three-sheets multimaterials spot welded assemblies. A simple spot weld connector model has been proposed. It is a macro modeling for applications to structural crashworthiness FEA. The parameters of this model have been identified using the experimental results of the proposed set-up in this work, and good results have been obtained.

This research is conducted through collaboration between the University of Valenciennes and the National Engineering School of Sfax. This collaboration is jointly financed in the frame of the Utique CMCU programme. The present research work has also been supported by the International Campus on Safety and Intermodality in Transportation, the Nord-Pas-de-Calais region, the European Community, the Regional Delegation for Research and Technology, the French National Research Agency, and by the Ministry of Higher Education and Research. The authors gratefully acknowledge the support of these institutions.

\section{References}

[1] Hartmann, E., Welding Journal (1958).

[2] VandenBossche, D. J., SAE paper 770214 (1977).

[3] Markiewicz, E., Drazetic, P., Mécanique et Industries 4, 17-27 (2003).
[4] Lee, Y., Wehner, T., Lu, M., Morrissett, T., Pakalnins, E., Journal of Testing and Evaluation 26, 213-219 (1998).

[5] Lin, SH., Pan, J., Tyan, J., Prasad, P., International Journal of Solids and Structures 40, 5539-64 (2003).

[6] Langrand, B., Combescure, A., International Journal of Solids and Structures 41, 6631-6646 (2004).

[7] Langrand, B., Markiewicz, E., International Journal of Impact Engineering 37, 792-805 (2010).

[8] Song, J.H. Huh, H. International Journal of Mechanical Sciences 53, 513-525 (2011).

[9] Langrand, B., Markiewicz, E., Drazetic, P., Geoffroy, P., Petitniot, J., Fabis, J., International Journal of Materials and Product Technology 12, 428-446 (1997).

[10] Langrand, B., Geoffroy, P., Petitniot, J., Fabis, J., Markiewicz, E., Drazetic, P., Aerospace Science and Technology 3, 215-227 (1999).

[11] Markiewicz, E., Ducrocq, P., Drazetic, P., Haugou, G., Fourmentraux, T., Berard, J.Y., International Journal of Materials and Product Technology 16, 484-509 (2001).

[12] Langrand, B., Deletombe, E., Markiewicz, E., Drazetic, P., Finite Elements in Analysis and Design 38, 21-44 (2001).

[13] Combescure, A., Delcroix, F., Caplain, L., Espanol, S., Eliot, P., International Journal of Impact Engineering 28, 783-802 (2003).

[14] Yang, X., Xia, Y., Zhou, Q., Engineering Fracture Mechanics 77, 1224-1239 (2010).

[15] Khandokera, N., Taklab, M., Materials and Design 54, 323-3304 (2014). 